\title{
Characteristic Signal of FT3 Measuring Probe
}

Jiri Vit ${ }^{1}$, Martin Novak ${ }^{2}$

${ }^{1}$ Hommel CS, Karoliny Svetle 2546, 41501 Teplice. Czech Republic. E-mail: jiri.vit@hommel-etamic.cz

${ }^{2}$ Faculty of Mechanical Engineering, J. E. Purkyne University in Usti nad Labem. Pasteurova 3334/7, 40001 Usti nad

Labem. Czech Republic. E-mail: martin.novak1@fvtm.ujep.cz

The article deals with the determining of the characteristic signal of the inductive FT3 measuring probe, that is used on the roundness machines of Jenoptik IM producer. The research was the basis for generalized recommendations for the calibration of the probe, which was published in the article "A roundness machine measuring probe calibration". The signals of eight FT3 probes have been acquired and evaluated for repeatability and mutual equality. Five signals of each individual probe were taken immediately for repeatability evaluation. The deviations of the individual signals from the mean signal and the deviations of the signal first derivative curves from the mean first derivative curve have been evaluated for the repeatability evaluation. The deviations of the signal of the individual probes from the perfect signal have been evaluated for the equality evaluation.

Keywords: Matlab, probe signal, roundness machine

\section{Introduction}

The generalized recommendations for the calibration of the FT3 probe, which is used on the roundness machines of Jenoptik IM producer, were published in the article [1]. The published recommendations were based on the analysis of its characteristic signal. This characteristic signal was chosen after reviewing of eight, in Czech Republic available, probes. The signals of each individual probe were evaluated from the view of the repeatability and the signals of all probes were compared from the view of the uniformity. The acquiring of the signal has been described in article [1] already. Procedures for the signal exploration [2] was built in Matlab. The repeatability was checked by the evaluation of five immediately successive signal of one probe. The deviations of the calibrated individual signals from the mean signal and the deviations of the individual first derivative curve from the mean curve of the first derivative curves were compared for the repeatability evaluation. The deviations of the calibrated signal and the perfect signal of the individual probes were compared for the uniformity evaluation.

\section{Terms definition}

The following terms have been introduced to evaluate the probe signal:

\section{Perfect signal}

The perfect signal has to be linear dependent on the touch point movement. It is expressed as a direction and an origin of the straight line according (1) in this case.

$$
y_{n}(i)=k\left(i-i_{0}\right) d x, k=1, i=1 \ldots n
$$

Where:

$\mathrm{n}$... the number of points in the profile $\left\{\mathrm{y}_{0}\right\}$

$i_{0} \ldots$ the index of the point of the profile where the beginning of the nominal signal straight line is located

$i$... the index of the point of the profile, for which the value of the perfect signal is calculated

$\mathrm{k}$... the direction of the straight line of the perfect signal

$\mathrm{dx}$... the spacing of the points of the profile $y_{n}(i) \ldots$ the value of the perfect signal at the $\mathrm{i}$-th point

\section{Filtered signal}

The filtered signal is a filtered mean line of the probe original signal. The Gaussian filter according [ (3)] with cut-off of $\lambda c=1 / 12$ of the profile length is used for the shortwave components removing in this case. The endeffects of the Gaussian filter are suppressed by the linear extrapolation.

\section{Calibrated signal}

The calibrated signal is a filtered signal adjusted so that the endpoint connecting line of the specified signal section has the same slope as the nominal signal. The length of the section corresponds to the value of the calibration standard and the section position corresponds to the target position of the contact run during the calibration. All signals have been calibrated in the procedure [2] so that the calibrated section lies between $-40 \mu \mathrm{m}$ and 0 $\mu \mathrm{m}$. The calibrated signal is described with (2)

Where:

$$
\mathbf{w}=\left[\mathbf{y}_{1}, \mathbf{y}_{2} \ldots \mathbf{y}_{\mathbf{n}}\right]
$$

$\mathrm{n}$... the number of points in the profile

$\mathbf{w}$... the line vector of the calibrated signal

\section{Deviation of the calibrated signal from the perfect} signal

The line vector according (3) and (4) providing information on the similarity of signals from individual probes being compared.

$$
\begin{gathered}
\delta=\left[\delta_{1}, \delta_{2} \ldots \delta_{i} \ldots \delta_{n}\right] \\
\delta_{i}=y_{i}-y_{n}(i), i=1 \ldots n \\
\delta_{\max }=\max _{i=1 \ldots n}\left(\operatorname{abs}\left(\delta_{i}\right)\right)
\end{gathered}
$$

Where:

$\mathrm{n}$... the number of points in the profile (2)

$y_{i} \ldots$ the $i$-th point of the calibrated signal according

$y_{n}(i) \ldots$ the perfect signal value at the $i$-th point of the profile according (1)

$\boldsymbol{\delta}$... the line vector of the deviations of the calibrated signal from the perfect signal 
$\delta_{\max } \ldots$ the maximal deviation of the calibrated signal from the perfect signal

\section{Mean calibrated signal}

The line vector according (6). Each point of the mean calibrated signal is the mean value of the corresponding points of the individual calibrated signals according (7).

$$
\begin{gathered}
\bar{w}=\left[\overline{\mathbf{y}}_{1}, \overline{\mathbf{y}}_{2} \ldots \overline{\mathbf{y}}_{\mathbf{i}} \ldots \overline{\mathbf{y}}_{\mathbf{n}}\right] \\
\overline{\mathbf{y}}_{\mathbf{i}}=\frac{1}{\mathrm{~m}} \sum_{\mathbf{j}=1}^{\mathrm{m}} \mathbf{y}_{\mathbf{i}, \mathbf{j}}, \mathbf{i}=\mathbf{1} \ldots \mathbf{n}
\end{gathered}
$$

Where:

$n$... the number of points in the profile

$\mathrm{m}$... the number of the profiles

$y_{i, j} \ldots$ the $i$-th point of the $j$-th calibrated signal according (2)

$\overline{\mathbf{w}}$... the line vector of the mean signal of all individual calibrated signals

\section{Deviation of the calibrated signal from the mean sig- nal}

The line vector according (8). Each point of the curve of deviations is the difference of the corresponding points of the relevant signal (1) and the mean signal (2) according (9).

$$
\begin{gathered}
\Delta \mathbf{w}=\left[\Delta \mathbf{y}_{1}, \Delta \mathbf{y}_{2}, \ldots, \Delta \mathbf{y}_{\mathbf{n}}\right] \\
\Delta \mathbf{y}_{\mathbf{i}}=\mathbf{y}_{\mathbf{i}}-\overline{\mathbf{y}}_{\mathbf{i}}, \mathbf{i}=\mathbf{1} \ldots \mathbf{n} \\
\Delta \mathbf{y}_{\max }=\max _{\mathbf{i}=\mathbf{1} \ldots \mathbf{n}}\left(\operatorname{abs}\left(\Delta \mathbf{y}_{\mathbf{i}}\right)\right)
\end{gathered}
$$

Where: (2)

$\mathrm{n}$... the number of points in the profile

$y_{i} \ldots$ the $i$-th point of the calibrated signal according

$\bar{y}_{i} \ldots$ the $i-t h$ point of the mean calibrated signal according (7)

$\Delta \mathbf{w} \ldots$ the line vector of the deviations of the individual calibrated signal from the mean signal

$\Delta y_{\max } \ldots$ the maximal deviation of the individual calibrated signal from the mean signal

\section{First derivative of the calibrated signal}

The line vector of the directions of the calibrated signal curve between two consecutive points according (11) a (12).

$$
\begin{gathered}
w^{\prime}=\left[d y_{1}, d y_{2} \ldots d y_{i} \ldots d y_{n-1}\right] \\
d y_{i}=\frac{y_{i+1}-y_{i}}{d x}
\end{gathered}
$$

Where:

$n$... the number of points in the profile

(2)

$y_{i} \ldots$ the $i$-th point of the calibrated signal according

$\mathrm{dx}$... the spacing of the points of the profile

$\mathbf{w}^{\prime} \ldots$ the line vector of the first derivative curve

\section{Mean curve of the first derivative curves}

The line vector according (13). Each point of the mean curve is the mean value of the corresponding points of the individual first derivative curves according (14).

$$
\begin{aligned}
\bar{w}^{\prime} & =\left[\overline{d y}_{1}, \overline{d y}_{2} \ldots \overline{d y}_{i} \ldots \overline{d y}_{n-1}\right] \\
\overline{d y}_{i} & =\frac{1}{m} \sum_{j=1}^{m} d y_{i, j}, i=1 \ldots n-1
\end{aligned}
$$

Where:

$n$... the number of points in the profile $\mathrm{m}$... the number of the profiles

$\mathrm{dy}_{\mathrm{i}, \mathrm{j}} \ldots$ the $\mathrm{i}$-th point of the $\mathrm{j}$-th calibrated signal according (12)

$\overline{\mathbf{w}^{\prime}} \ldots$ the line vector of the mean curve of the first derivative curves

\section{Deviation of the first derivative curve from the mean curve of the first derivative curves}

The line vector according (15) providing information on the equality of individual first derivative curves and consequently on the equality of individual signals of the probe. Each point of the curve of the deviations is the difference of the corresponding points of the relevant first derivative curve and the mean curve of the first derivative curves according (16).

$$
\begin{gathered}
\Delta w^{\prime}=\left[\Delta d y_{1}, \Delta d y_{2}, \ldots \Delta d y_{i} \ldots, \Delta d y_{n-1}\right] \\
\Delta d y_{i}=d y_{i}-\overline{d y}_{i}, i=1 \ldots n-1 \\
\Delta d y_{\max }=\max _{i=1 \ldots n}\left(\operatorname{abs}\left(\Delta d y_{i}\right)\right)
\end{gathered}
$$

Where:

$\mathrm{n}$... the number of points in the profile (12)

$\mathrm{dy}_{\mathrm{i}} \ldots$ the $\mathrm{i}$-th point of first derivative curve according

$\overline{\mathrm{dy}}_{\mathrm{i}} \ldots$ the $\mathrm{i}$-th point of the first derivative mean curve according (14)

$\Delta \mathbf{w}^{\prime} \ldots$ the line vector of the deviations of the individual first derivative curve from the mean curve

$\Delta \mathrm{dy}_{\max } \ldots$ the maximal deviation of the individual first derivative curve from the mean curve

\section{Waviness of the first derivative curve of the cali- brated signal}

The line vector according (19) containing the deviations of the first derivative curve according (11) and its filtered mean line according (18). In metrology of surfaces, the profile free of shortwave components is called the waviness profile, and therefore this term is also used here. The waviness provides the information on the smoothness of the signal curve. The spline filter according [4] with cut-off of $\lambda c=1 / 5$ of the profile length is used for the shortwave components removing in this case.

$$
\begin{gathered}
\widehat{\mathbf{w}}=\left[\hat{\mathbf{y}}_{1}, \hat{\mathbf{y}}_{2} \ldots \hat{\mathbf{y}}_{\mathbf{i}} \ldots \hat{\mathbf{y}}_{\mathbf{n}-1}\right] \\
\Delta \widehat{\mathbf{w}}=\left[\Delta \hat{\mathbf{y}}_{1}, \Delta \hat{\mathbf{y}}_{2} \ldots \Delta \hat{\mathbf{y}}_{\mathbf{i}} \ldots \Delta \hat{\mathbf{y}}_{\mathbf{n}-1}\right] \\
\Delta \hat{\mathbf{y}}_{\mathbf{i}}=\mathbf{d y}_{\mathbf{i}}-\hat{\mathbf{y}}_{\mathbf{i}}, \mathbf{i}=\mathbf{1} \ldots \mathbf{n}-\mathbf{1} \\
\Delta \hat{\mathbf{y}}_{\max }=\max _{\mathbf{i}=\mathbf{1} \ldots \mathbf{n}}\left(\operatorname{abs}\left(\Delta \hat{\mathbf{y}}_{\mathbf{i}}\right)\right)
\end{gathered}
$$

Where:

$\mathrm{n}$... the number of points in the profile (12)

$\mathrm{dy}_{\mathrm{i}} \ldots$ the $\mathrm{i}$-th point of first derivative curve according

$\widehat{\mathbf{w}}$... the line vector of the filtered mean line of the first derivative curve

$\hat{y}_{i} \ldots$ the $i$-th point of filtered mean line of the first derivative curve

$\Delta \widehat{\mathbf{w}} \ldots$ the line vector of the deviations of the first derivative curve from its mean line

$\Delta \hat{y}_{\max } \ldots$ waviness - the maximal deviation of the first derivative curve from its mean line 


\section{Graphical output}

The procedures [2] for the probe signal exploration provide various graphical outputs. In this case have been used the charts according Fig. 1 and Fig. 2.

The Fig. 1 shows the series of the signals which were taken immediately in succession out of one probe.

- The upper left quadrant displays graphs of the deviations of the calibrated signals from the nominal signal according (3). The calibrated section from $-40.00 \mu \mathrm{m}$ to $0.00 \mu \mathrm{m}$ of the probe range is in alignment with the horizontal axis representing the nominal signal.

- The upper right quadrant displays graphs of the deviations of the calibrated signals from
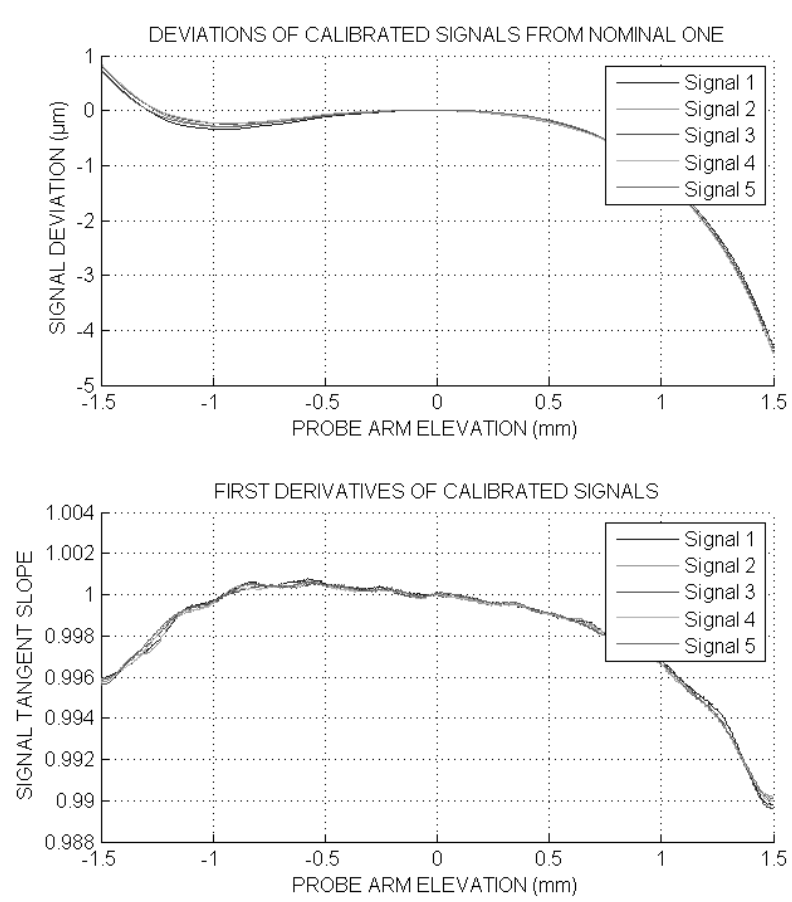

Fig. 1 Output charts for a series of signals

The Fig.2 shows the curves of only one signal.

- The left side displays the first derivative curve (blue thin line) according (11) and its filtered mean line (red bold line) according (18).

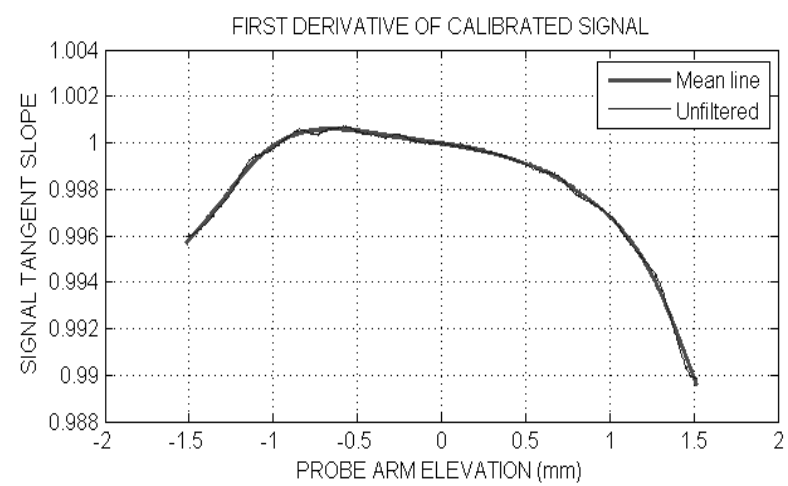

the mean signal according (8). Every individual signal is in alignment with the mean signal in the calibrated section from $-40.00 \mu \mathrm{m}$ to $0.00 \mu \mathrm{m}$ of the probe range.

- The lower left quadrant displays the curves of the first derivatives according (11). The calibrated section from $-40.00 \mu \mathrm{m}$ to $0.00 \mu \mathrm{m}$ of the probe range has the value of the first derivative equal to one.

- The lower right quadrant displays the curves of the deviations of the first derivatives from the mean curve of the first derivatives according (15).
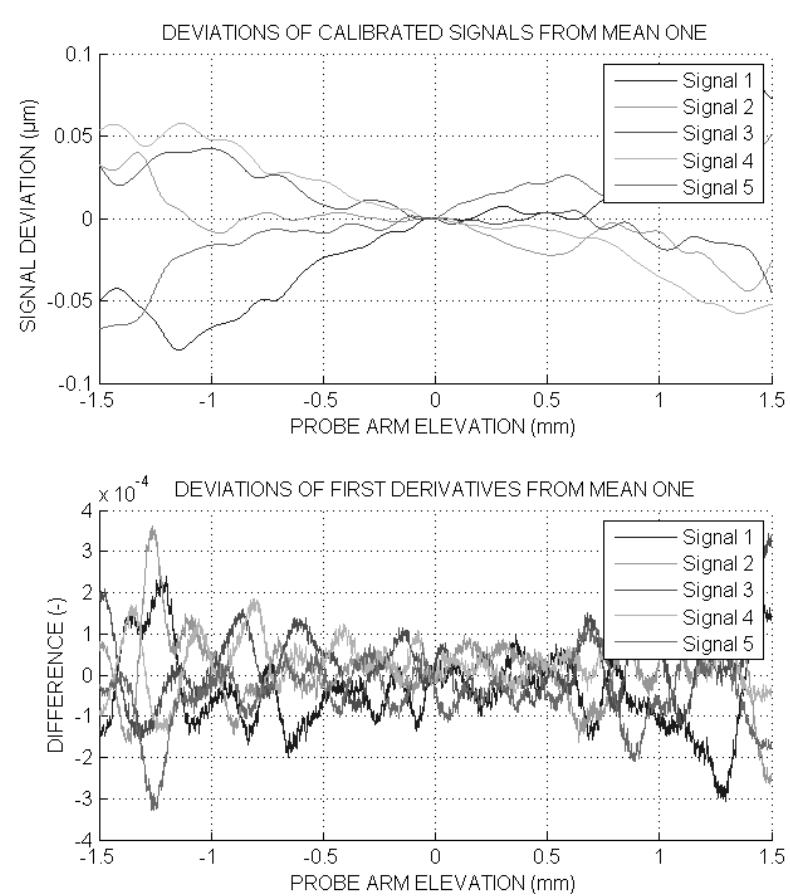

Fig. 2 Output charts for single signal

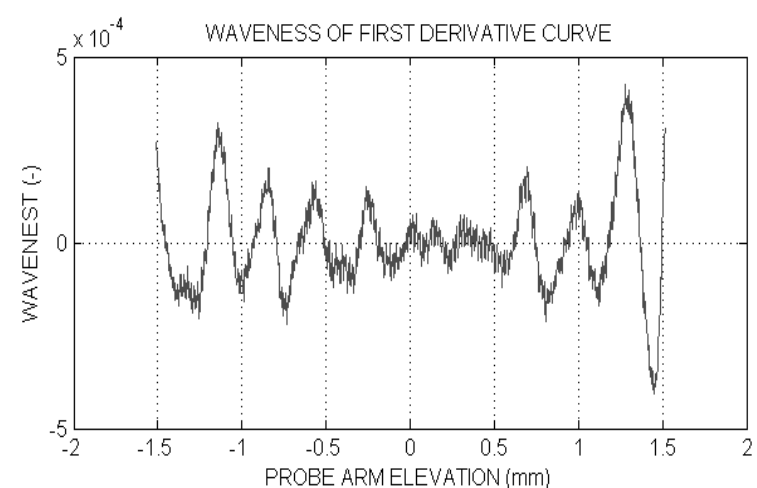

- The right side displays the deviation of the first derivative curve from its filtered mean line according (19). 


\section{Numerical results}

The results have been calculated for eight probes. Each probe is identified with a serial number, see Fig.3. Five signals have been taken immediately from each probe.

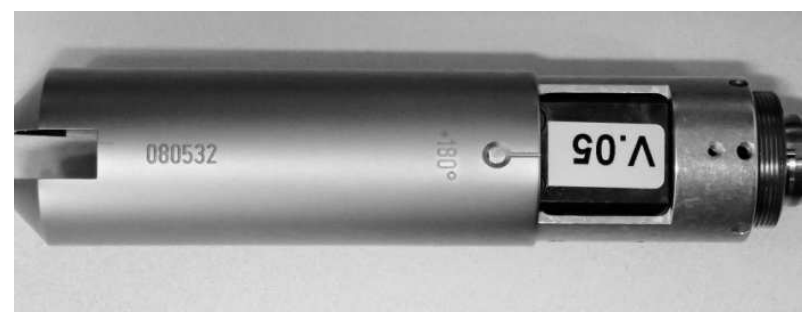

Fig. 3 Measuring probe FT3

The results are displayed at the table Tab. 1. Every value is the maximal value of five signals of one probe: $\delta_{\max 5} \ldots$ the maximal value of $\delta_{\max }$ of five signals of one probe. The $\delta_{\max }$ is the maximal deviation of the calibrated signal from the perfect signal according (5).

$\Delta y_{\max 5} \ldots$ the maximal value of $\Delta y_{\max }$ of five signals of one probe. The $\Delta y_{\max }$ is the maximal deviation of the individual calibrated signal from the mean signal according (10).

$\Delta \mathrm{dy}_{\max 5} \ldots$ the maximal value of $\Delta \mathrm{dy}_{\max }$ of five signals of one probe. The $\Delta \mathrm{dy}_{\max }$ is the maximal deviation of the individual first derivative curve from the mean curve according (17).

$\Delta \hat{y}_{\max 5} \ldots$ the maximal value $\Delta \hat{y}_{\max }$ of five signals of one probe. The $\Delta \hat{y}_{\max }$ is the maximal deviation of the first derivative curve from its mean line according (21).

Tab. 1 The characteristics of the signal of the probes

\begin{tabular}{|c||c|c|c|c|}
\hline Probe & $\delta_{\max 5}[\mu \mathrm{m}]$ & $\Delta y_{\max 5}[\mu \mathrm{m}]$ & $\Delta d y_{\max 5}[-]$ & $\Delta \hat{y}_{\max 5}[-]$ \\
\hline \hline S/N 080512 & 4.425 & 0.086 & $3.62 \times 10^{-4}$ & $8.33 \times 10^{-4}$ \\
\hline S/N 080532 & 2.659 & 0.317 & $5.07 \times 10^{-4}$ & $7.65 \times 10^{-4}$ \\
\hline S/N 080610 & 3.310 & 0.145 & $4.54 \times 10^{-4}$ & $8.58 \times 10^{-4}$ \\
\hline S/N 135017 & 2.432 & 0.148 & $3.32 \times 10^{-4}$ & $8.60 \times 10^{-4}$ \\
\hline S/N 080585 & 2.740 & 0.233 & $8.07 \times 10^{-4}$ & $12.28 \times 10^{-4}$ \\
\hline S/N 135076 & 4.963 & 0.251 & $3.19 \times 10^{-4}$ & $7.64 \times 10^{-4}$ \\
\hline S/N 128077 & 2.993 & 0.191 & $2.81 \times 10^{-4}$ & $7.25 \times 10^{-4}$ \\
\hline S/N 100731 & 2.570 & 0.194 & $3.98 \times 10^{-4}$ & $6.84 \times 10^{-4}$ \\
\hline
\end{tabular}

\section{Conclusion}

The FT3 probe is equipped with a friction clutch for manual tilting of the arm and does not allow the arm position to be locked. The actual position depends on the skill of the operator and is probably not the same for all probes. Different probe arm position causes the different arc error. Therefore, the deviations of the calibrated signal from the perfect signal do not have much importance in deciding about signal uniformity.

The first derivative curves of the signals of one probe show similar unevennesses, the cause of which is likely the electrical-kinematic characteristic of the probe. The first derivative curves are only similar even in calibrated section, due to external disturbance. This is why the separately in software calibrated individual signals of one probe have little bit different direction and why the deviation of the individual calibrated signal from the mean signal increases towards ends.

From this perspective, the evaluated signals are very similar except for the signal of the probe of S/N 080585. For further making of calibration recommendations, the signal of the probe of S/N 080532 will be used as a characteristic signal.

\section{References}

[1] VÍT, J., NOVÁK, M. (2018) A roundness machine probe calibration. In Manufacturing Technology, Vol. 18, No. 6, pp. 1053-1059. ISSN 1213-2489.

[2] VÍT, J. (2017) Procedure library for roundness machine probe signal exploration. Usti nad Labem, 2017. Study for dissertation theses. Faculty of Mechanical Engineering, J. E. Purkyne University. Supervisor Martin Novak

[3] - - Surface metrology open profile Gaussian filter. Library of procedures. [online]. 06. 092015. Matlab Central File Exchange. [Citation: 18. 10 2018.] Available on: http://www.mathworks.com/matlabcentral/fileexchange/52892-surface-metrology-open-profilegaussian-filter.

[4] _. Surface metrology open profile spline filter. Library of procedures. [online]. 19. 082015 Matlab Central File Exchange. [Citation: 18. 10 2018.] Available on: http://www.mathworks.com/matlabcentral/fileexchange/52562-surface-metrology-open-profile-spline-filter. 